\title{
JUEGO Y PSICOMOTRIC IDAD Propuesta y análisis de un programa de trabajo (Segunda parte)
}

\section{Autores} C.V.O. Consejería para la Igualdad y Bienestar Social
Dr. Juan Miguel Arráez Martínez Facultad de Ciencias de la Educación Universidad de Granada

\section{Resumen}

Jugar es para todo niño una necesidad esencial y la herramienta que le permitirá conocerse a sí mismo y el mundo al que pertenece. La psicomotricidad propone un modelo diferente de acercarse al niño desde el respeto a sus necesidades, su manera de ser y de expresarse.

Partiendo de esta premisa se ha intentado fundamentar la intervención psicomotriz que se ha seguido con un niño de 20 meses de edad, diagnosticado con Síndrome de Down, que acude al centro en el que realizo mi actividad profesional.

Se analizan las actividades y situaciones lúdicas que se han favorecido durante la intervención y se concluye: 1) Confirmando la psicomotricidad como metodología eficaz en el planteamiento de objetivos y éxito de logros; 2) Destacando la importancia de contar con espacios, materiales y formación específica para intervenir en estas edades; 3) Considerando que los juegos descritos (sensoriomotores, simbólicos y de representación) promueven la madurez del niño y favorecen la adquisición de competencias y estrategias adecuadas para su desarrollo y 4) Subrayando el valor de la interacción afectiva con el adulto a partir de la propuesta de situaciones de reaseguración profunda y de maternaje.

Palabras clave: Juego infantil, Desarrollo lúdico. Psicomotricidad. Metodología psicomotriz dinámica. Atención a la diversidad, Síndrome de Down...

\section{Introducción}

En la primera parte de este documento se ha justificado la importancia que tienen las actividades lúdicas infantiles en el desarrollo integral del niño y se ha fundamentado la metodología psicomotriz de corte dinámico a utilizar. En esta segunda parte se define, registra e interpreta la intervención que, partiendo de dichos presupuestos, se lleva a cabo con un sujeto de 20 meses diagnosticado de síndrome de Down. Se propone el programa de trabajo, se recoge una sesión "tipo", se describen los juegos realizados a lo largo del todo el proceso que duró la intervención y se apuntan una serie de conclusiones relevantes que se derivan de la actividad realizada y que justifican la actuación efectuada así como la línea de investigación que estamos desarrollando en al ámbito de la discapacidad.

\section{Un caso práctico: José}

José (nombre figurado), a petición de sus padres y orientados por el médico de familia de su pueblo, acude a nuestro centro con el objetivo de recibir asistencia. Una vez superado el protocolo de valoración que se le realiza es orientado al área de Psicomotricidad en la que se le acoge y donde se le asignan dos sesiones semanales.

\section{Datos personales:}

José tiene 20 meses cuando se realiza su primera visita y entrevista en el centro. Es hijo único de una pareja sana en la que hay gran diferencia de edad entre los cónyuges. Proceden de un entorno rural en el que viven desahogadamente con el negocio que regenta el padre.

Fruto de un embarazo deseado, fue un parto espontáneo y a término sin ninguna complicación. 
José respondía a un fenotipo síndrome de Down que en estudio genético se confirmó días más tarde. No precisó de intervención hospitalaria aún cuando se registró una hipotonía global con movilidad libre de miembros superiores e inferiores y actitudes corporales normales.

Sus padres lo describen como un niño tranquilo, llora cuando algo le molesta pero se calma con facilidad tras cogerlo en brazos, acunarlo y ponerle chupete. En el momento de su llegada al centro no va a ninguna guardería, aunque ahora sus padres se lo están planteando. Ha estado asistiendo a diversos gabinetes de estimulación de su localidad por consejo de su pediatra.

En casa está bien atendido y aceptado por el resto de la familia extensa (abuelos, primos, tíos...). Se entretiene poco solo y prefiere llamar la atención de su madre. Muestra interés por estímulos visuales (televisión) aunque con tiempo de atención muy limitado. No plantea problemas extraordinarios en la rutina diaria, se le regaña cuando algo no está bien y se le recompensa con besos, "arrumacos" y palabras de cariño. Reconocen que son muy permisivos con él y que está algo consentido.

Al aplicar la Escala para Medir el Desarrollo de la Primera Infancia: BRUNET-LEZINE (1971) se obtiene un Cociente de Desarrollo de 0'77, que se corresponde con una edad de desarrollo de 14 '1m. En términos generales se aprecia cierto desajuste en todas las áreas siendo más pronunciado en la categoría de lenguaje que queda muy por debajo de la media; las áreas motora, social y cognitiva también se encuentran afectadas, pero algo menos.

En las primeras situaciones y observaciones que se realizan en sala se descubre a un niño alegre, algo disperso y muy interesado por todo aquello que se lo ofrece. Maneja los objetos con seguridad (manipula objetos de diferentes tamaños, los introduce en botes voluntariamente y por orden e incluso inicia construcciones sencillas colocando un taco sobre otro), de un modo creativo (se interesa por botes con ranuras y agujeros $e$ investiga autónomamente la manera de introducir objetos en ellos) y con capacidad para mantener el juego que se le propone e incluso de iniciar algunos muy básicos él mismo. Se mueve por la sala de un modo coordinado bien reptando o gateando y cuando llega a un lugar que le interesa se sienta y manipula aquello que ha conseguido. Está atento a sus padres a los que requiere con la mirada pero acepta de buen grado la presencia y las intervenciones de un adulto extraño -el psicomotricista- que le retira y ofrece diferentes elementos.

Tiene adquiridos algunos patrones motores, los utiliza de un modo adecuado en sus desplazamientos y está iniciándose en otros más complejos: ponerse de pie solo, subir las escaleras, subir y bajar rampas por las que se desplaza deslizándose y gateando sin miedo. Manipula con ambas manos manteniendo un objeto en cada una de ellas; realiza golpeteos en vertical y horizontal, usa la pinza, aún de un modo vasto, y comienza a utilizar el dedo índice en la exploración de agujeros y demás orificios. Mete y saca por orden y usa preferentemente su mano derecha aunque sin dominancia establecida.

Atiende y se interesa por dibujos y cuentos siguiendo una historia hasta el final. Mantiene la atención centrada y durante bastante tiempo si lo que está haciendo le interesa. Es capaz de descubrir objetos que han sido escondidos ante su mirada, así como de resolver otros problemas lógicos básicos que se le proponen (tirar de cuerda para atraer objetos, levantar un bote para descubrir una canica escondida...).

Comprende y sigue órdenes sencillas: "toma" y "dame" y con apoyo gestual es capaz de seguir otras que incluyen lugares y posiciones. Mantiene un repertorio verbal básico (sobre todo con sus padres) en el que se incluyen algunas onomatopeyas y sonidos de animales que reconoce y reproduce. Repite todo aquello que sus padres le dicen y responde con gestos a la orden de despedida, saludo, etc. Llama verbal y gestualmente y de diferente modo a sus tíos, abuelos y al perro de sus vecinos.

\section{Propuesta de trabajo}

Siguiendo una metodología psicomotriz que se enmarca en la tradición dinámica de Aucouturier (Aucouturier, Darrault y Empinet, 1985; Arnaiz, 2000; Llorca, 2002), partiendo del respeto al momento evolutivo en el que se encuentra José y a los intereses y demás características personales que se deducen de las sesiones de observación, se propone un programa de trabajo que se irá desvelando poco a poco a sus padres en sucesivas reuniones que se mantendrá con ellos. En una primera etapa del tratamiento los padres participarán directamente en las sesiones, más adelante se les invitará a quedarse fuera aunque se les informará diariamente tanto de las conquistas y nuevas adquisiciones que se vayan consiguiendo como de las dificultades que se descubran, además de todo aquello que se estime conveniente y esté relacionado con el tratamiento que se realiza con su hijo.

El programa es orientativo y está abierto a los cambios que haya que realizar en función de los progresos terapéuticos que se vayan observando. 
Para mejorar sus habilidades motoras se propone: introducir nuevas destrezas (bipedestación, buen apoyo plantar, pasos laterales con ayuda, marcha autónoma) y perfeccionar aquellas otras que ya están presentes introduciendo fuerza y calidad en esas conductas; mejorar el tono y evitar disociación de movimientos estableciendo secuencias lógicas encadenadas; desarrollar el control y el dominio de la postura; presentar situaciones motrices que ha de resolver y que requieren aplicación de estrategias que implican cambios posturales, animándole a que lo haga del modo más armónico y con el mayor placer posible.

En cuanto al área manipulativa interesa mejorar su forma de asir los objetos (mantener varios objetos en una mano, pasar hábilmente de una a otra mano...); iniciar actividades que requieren destrezas y control manipulativo (construcción de torres con cubos, desarmar juguetes, abrir caja de diferentes formas y tamaños para descubrir lo que hay en su interior...); introducir el garabateo e incorporar otras actividades en las que se pone de manifiesto la utilidad de su mano (arrugar papel, desenvolver objetos, pasar páginas de un libro...).

Para optimizar el aspecto perceptivo-cognitivo se presentarán problemas lógicos más complejos (juegos de memoria en los que ha de recordar en cual de las tazas -dos o tres-que se le muestran ha sido escondido un objeto, dar un rodeo para buscar objetos que desaparecen...): se ampliará el repertorio de juegos imitativos sobre diferentes partes del cuerpo (tocar la nariz, sacar la lengua, señalar los ojos, etc.); realización de construcciones sencillas sin modelo (tren con cubos, caminos con maderas...); se iniciarán las simbolizaciones con y sin apoyo de objetos reales; se introducirán emparejamientos elementales entre objetos reales y objetos figurados y algo más adelante entre estos y dibujos de los mismos. Es también el momento de iniciar conceptualizaciones espacio-temporales (dentro/fuera, cerca/lejos)y de contenido lógico-matemático (uno/muchos).

En la atención de los aspectos comunicativos y de autoayuda se recibe apoyo de la logopeda del centro. Se provocarán situaciones en las que ha de responder a su nombre, imitar producciones que realice el adulto (onomatopeyas, soplo, comprensión de prohibiciones y demás órdenes con un componente verbal); se motivará la presencia de intención comunicativa en su expresión ampliando el repertorio de peticiones verbales (ir a un determinado lugar, alcanzar un objeto, ponerse los zapatos...) y asociaciones de palabra e imagen...

También se orientará a los padres en las cuestiones que se estimen necesarias relacionadas con los hábitos alimentarios, de aseo y sueño así como otros de carácter social adecuados a su edad (dependencia/independencia, escolarización, rabietas, etc.).

\section{Procedimiento: recogida de datos}

El tratamiento psicomotor seguido con José entre los meses de febrero a junio de 2003 ha sido recogido y analizado detalladamente. Durante el tiempo que duró la intervención se realizaron un total de 22 sesiones de trabajo.

Se ha seleccionado este caso de entre todos los que reciben tratamiento en el centro porque, siguiendo un criterio estratégico, se ha considerado el más idóneo y representativo de la población con la que se contaba: un sujeto que por edad y por su momento evolutivo aportaría bastante información acerca de la intención que se quería analizar en este documento: registrar y describir, sin intención de generalizar, toda actividad lúdica surgida durante el período que ha durado la intervención psicomotriz.

Todas las sesiones realizadas y una vez que concluían, eran registradas personalmente por escrito en una hoja de registro confeccionada a tal propósito, intentando recopilar el mayor número de datos y del modo más objetivo posible. En la página siguiente se trascribe una de ellas (aleatoriamente escogida) con la finalidad de exponer cómo es una sesión de trabajo estándar.

En esta hoja de registro se intenta que, diariamente, quede recogido el nombre y fecha de la sesión, la propuesta de trabajo (objetivos que se persiguen) y otros datos referidos a las actividades que se desea realizar, materiales con los que se trabaja y el desarrollo de la sesión. Se anota también el tiempo que permanece el niño en cada una de las actividades (sólo en el caso de ser una sesión grabada o que sea recogida por observadores externos) y la información que se da posteriormente a los padres. Se incluye además algunas conclusiones e hipótesis de trabajo y recomendaciones y orientaciones que puedan servir para futuras sesiones.

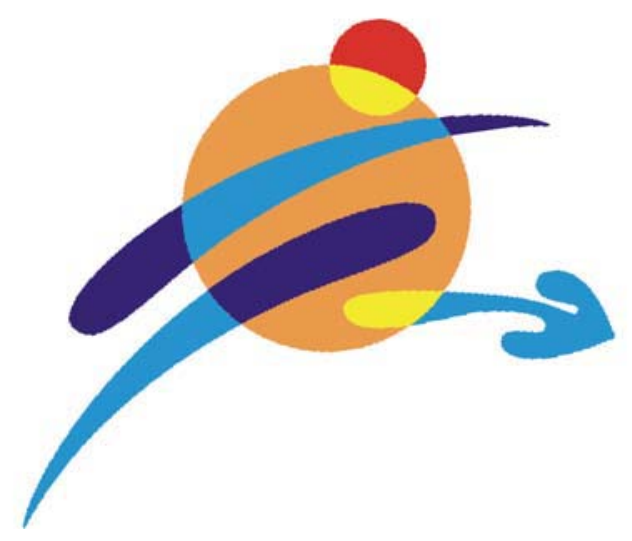




\section{PROPUESTA DE TRABAJO:}

M.G: Afianzar gateo y uso de patrón incorporado a desplazamientos.

Posición de bipedestación. Paso de gateo a bipedestación con apoyo. Desplazamiento en línea recta.

M.F: Dedo índice. Utilización. Presentación de pinza.

Preparar la sala para afianzar sobretodo el proceso motor de cambios posturales elementales.

PREPARAR PROGRAMA PARA LOS PADRES.

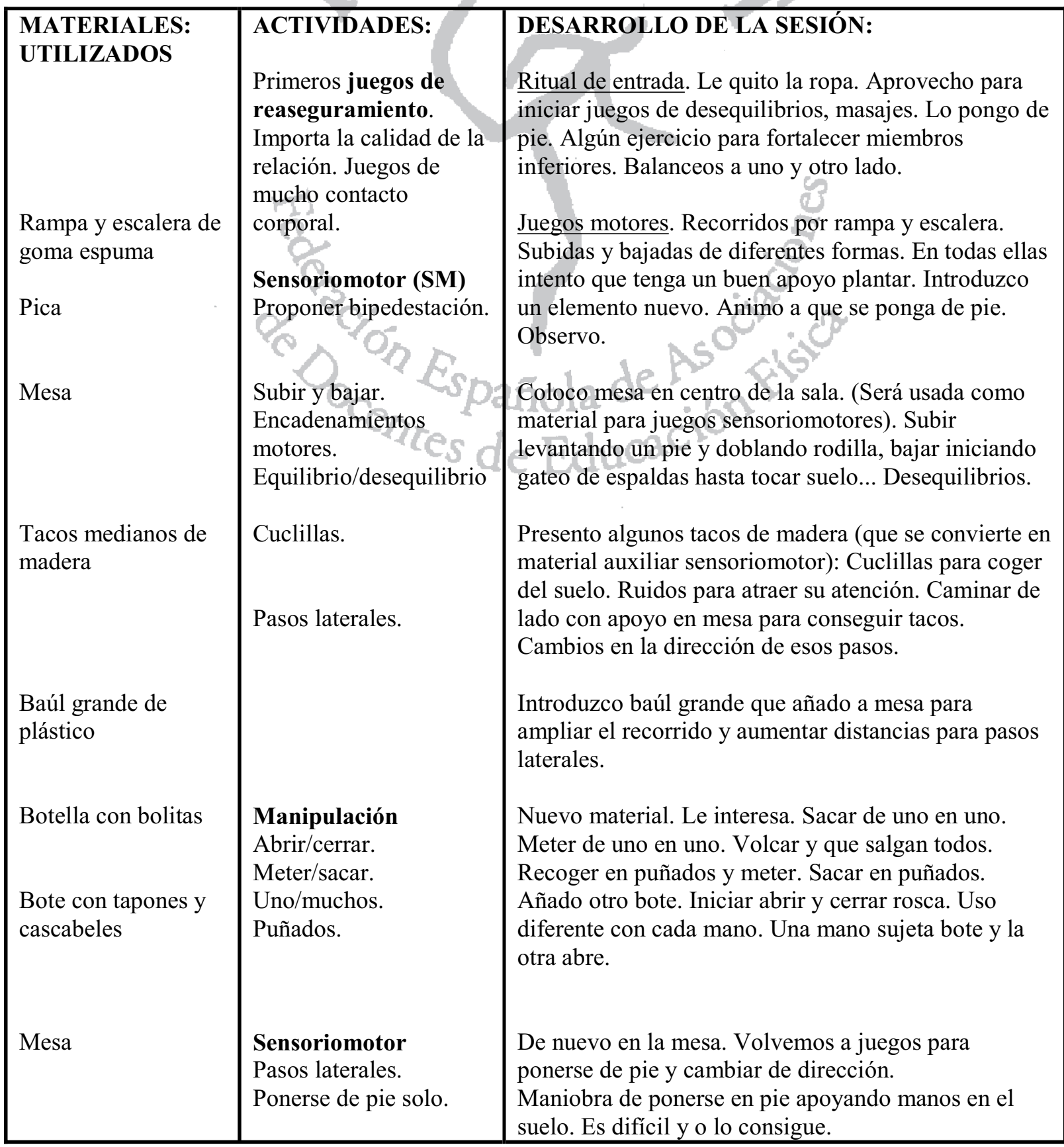




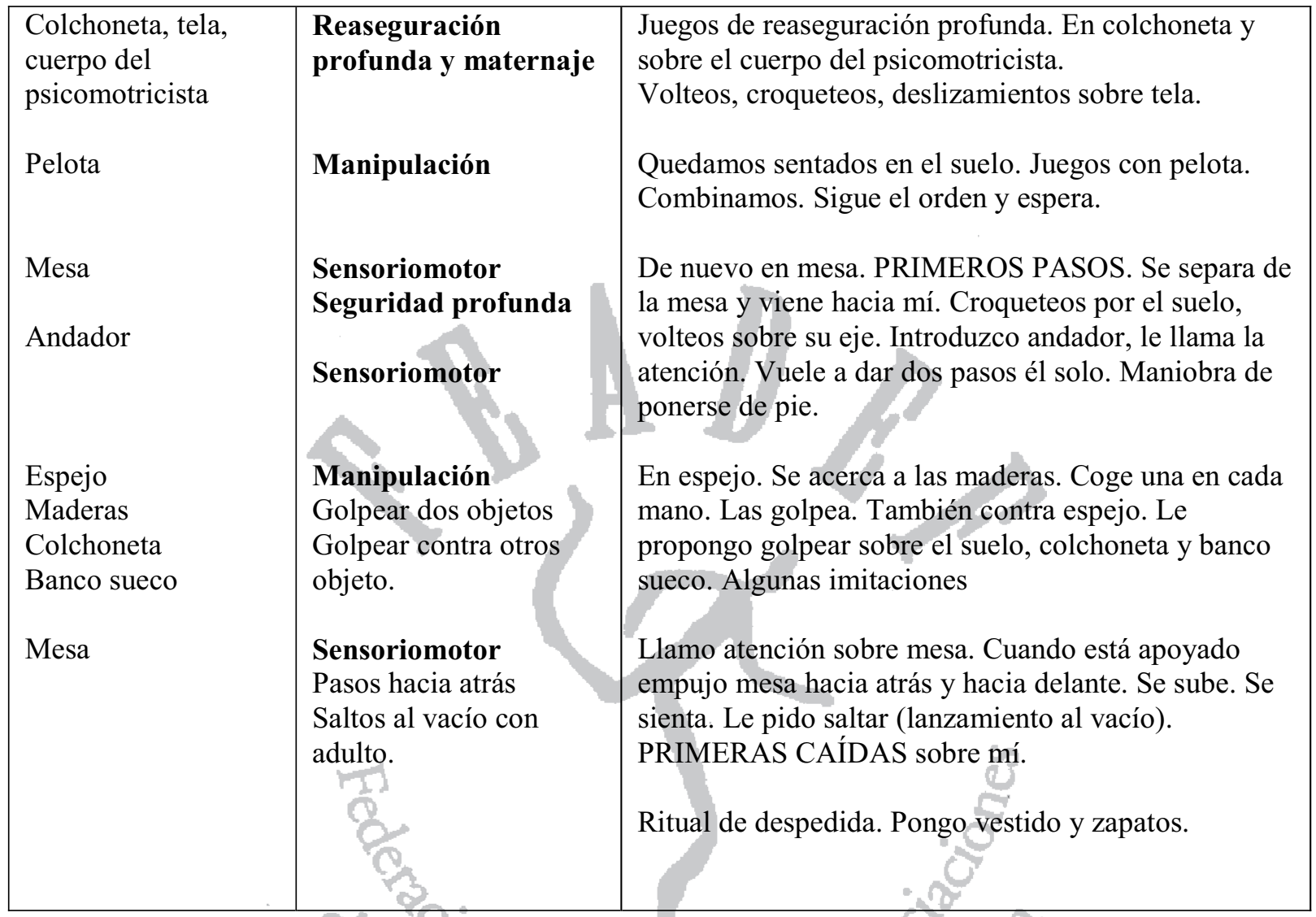

\section{COMENTARIOS:}

Sesiones muy productivas. Su nivel de atención es muy bueno y la sesión es muy fácil de realizar porque es posible orientar hacia el objetivo que se persigue.

\section{INFORMACIÓN A LOS PADRES:}

Comentamos la sesión. Hincapié en el tema del lenguaje: a) baño de lenguaje en todo lo que casa se hace con él y b) pedir que vaya produciendo (terminaciones de palabras y consolidar las identificaciones).

Hay que hablar del programa y actualizar. Ver que se está hace y consigue en casa.

\section{CONCLUSIONES/NOTAS:}

Atención al lenguaje de acción

Da sus primeros pasos. Fomentar en las próximas sesiones. Preparar más ejercicios a este respecto.

Inicio de caídas al vacío. Se siente muy seguro. Lo hace sin miedo. Fomentar sin riesgos. 


\section{Juegos sensoriomotores de José}

La sala de psicomotricidad está preparada, en el espacio denominado de juego sensoriomotor, con materiales agradables y blandos como colchonetas, bloques de goma espuma, telas, etc., para dar al niño la posibilidad de vivir motrizmente su cuerpo, el placer de crecer, esforzarse y hacer cosas cada vez más difíciles.

Ha interesado recoger todas las producciones que José ha realizado en la sala. Han sido anotadas en las hojas de registro y un posterior análisis nos ha permitido, de acuerdo con el tipo de actividad realizado, encuadrarlas en uno u otro de los grupos referenciados. Estas son las actividades que se han recogido:

\subsection{Juegos de seguridad profunda}

Deslizar por el suelo tumbado sobre telas y plásticos grandes.

Deslizar tirando suavemente por los pies, por las manos.

Realizar volteretas atrás y adelante sobre piernas del adulto, cogiéndole por los tobillos o las manos.

Croqueteos (giros sobre sí mismo) estando en colchoneta o sobre el cuerpo del psicomotricista.

Giros provocados por el adulto sobre la colchoneta, empujando desde diferentes partes de su cuerpo.

Estando sentado sobre mis piernas provocar desequilibrios, balanceos, caídas hacia uno y otro lado.

Arrastrar suavemente su cuerpo, subir o bajar por algunos de los bloques (rampas) preparados en la sala y dejar resbalar por dichos bloques. Introducir cambios posturales en la manera de realizar las subidas y bajadas (sentado, en decúbito prono, en decúbito supino).

Estando sobre la pelota BOBATH, realizar desequilibrios que le hagan realizar ajustes corporales autónomos y caídas controladas al suelo, reacciones de apoyo, etc.

Arrastrar, estando sentado sobre diferentes elementos: tela, colchoneta, pliego de cartón, cajas $u$ otros materiales y realizar recorridos por la sala. Introducir variaciones en la velocidad, los giros, frenado brusco, etc.

Voltear con seguridad y apoyo hacia uno y otro lado.
Subir por los aires, apoyando su pecho entre las piernas del psicomotricista e introducir variaciones en los movimientos de subida, bajada, hacia la derecha, izquierda, atrás, adelante, etc.

Sentar sobre mis piernas, dejar caer al suelo y rodearlo.

Estando en pie con apoyo, mover de uno a otro lado, incrementando la fuerza del movimiento hasta conseguir que quede sobre una sola pierna, que caiga sentado, que caiga sobre mí.

\subsection{Juegos de maternaje}

Acariciar y masajear cualquier parte de su cuerpo con las manos, con pelotas, con papel de seda, con telas, con globos, etc.

Jugar con sus manos, tirar de ellas suavemente, frotarlas, llevarlas a acariciar partes de su cuerpo y del mío.

Rodearlo con las telas grandes: envolverlo, hacer aire y caricias, etc. Tapar y destapar bruscamente y con suavidad.

Abrazar suavemente.

Dar palmadas suaves que recorren su espalda y otras partes de su cuerpo.

Cantar canción mientras acaricio su cara y otras partes de su cuerpo.

Tumbar sujetando su espalda con mi pecho e iniciar balanceos que se irán modificando en fuerza y velocidad.

Cosquillas (juegos de las hormigas que suben y bajan, manos como plumas que caen sobre su cuerpo, dedos como gotas de lluvia que van cayendo).

\subsection{Juegos presimbólicos - de contraste}

Vaciar cajas de golpe para ver qué hay en su interior.

Meter objetos groseramente (en puñados) dentro de una caja o de una lata.

Jugar a abrir y cerrar las manos, escondiendo cosas en su interior.

Repartir las bolitas que tiene en sus manos, reconociendo que da algo que le pertenece y quiere que se guarde como un tesoro.

Tapar y destapar con las telas grandes de colores. Descubrir dónde ha quedado escondido, llamarlo para que aparezca. Juego del cu-cu. 


\subsection{Juegos sensoriomotores (propiamente dichos)}

Atraer su atención con objetos y provocar desplazamientos por la sala que impliquen movimientos de gateo, arrastre, volteos, ponerse de pie, pasos laterales, etc.

Ayudar, empujando por los pies, a realizar desplazamientos sobre bloques de goma espuma 0 por el suelo de la sala.

Animar a recuperar objetos lanzados por la sala. Hacer que descienda solo por rampas para recuperar objetos que he lanzado.

Utilizar los diferentes bloques de goma espuma para provocar el mayor número posible de encadenamientos motores y cambios posturales.

Subir y bajar de forma autónoma por los diferentes elementos que hay en la sala.

Ampliar su repertorio motor: cuclillas, agacharse, sentarse en el suelo.

Ayudar a realizar maniobras concretas: patrón de bipedestación.

Ejercicios para eliminar posturas incorrectas: hiperextensión de rodillas, sentarse en W.

Dar vueltas estando sentado sobre nalgas, en la misma dirección que yo lo hago.

Perseguir gateando elementos que hay en la salay que yo voy cambiando de sitio aumentando dificultad: sobre escalera, encima de mesa, debajo de banco sueco, etc.

Iniciar maniobras especiales: subir a silla y sentarse, voltearse y bajar.

Bajar por la escalera de diferentes formas: sentado, en prono, en decúbito supino.

Realizar pasos estando sujeto con una tela que pongo alrededor de su pecho.

Rodar sobre cilindro de goma espuma. Cambiar dirección. Ir hacia atrás y adelante.

Fomentar reacciones de apoyo manual adecuadas.

Sentar sobre un cilindro con un píe a cada lado. Rodar en una y otra dirección. Fortalecer apoyo plantar.

Estrategias posturales para resolver situaciones más complejas: salir de cajas con apoyo en banco sueco y bloques.

Introducir nuevos elementos: triciclo. Avance con los pies. ¿Pedaleo?

Sobre pelota BOBATH. Balanceos, vaivenes, apoyo plantar. Inicio de caída al vacío, desequilibrios.

Pasar de un lado a otro de banco sueco y de diferentes formas posibles: de cabeza, avanzando pie, etc.

\subsection{Juegos simbólicos 0 de roles}

Reproducir acciones de la vida diaria: comer, peinarse, vestirse, lavarse las manos.

Dar de comer, vestir, peinar, dar biberón, etc. a una muñeca con la que está jugando.

Manifestar y exteriorizar sentimientos. Acariciar y balancear a una muñeca que tiene en brazos, castigar y pegar.

Preparar una cuna, balancear y dormir a una muñeca a la que ha dado de comer previamente.

Inventar usos distintos para el cajón de plástico: utilizar como un tren, como una cuna, como una casa. Realizar juegos en los que ese material tiene ese referente.

Reconocimiento e identificación de objetos de la caja de material simbólico: frutas, muñeca, biberón, peine, etc. Reproducción de acciones de la vida diaria.

Chocar unos tacos con otros como si se tratase de coches que circulan.

Juegos de espadas y luchas con las picas (juegos propuestos por él).

Imitar el montar a caballo sobre picas.

Construcciones con bloques, simulando una casa. Bloques que se apilan, con un significado concreto.

Utilizar las picas como bastones y andar por la sala con ellos.

Sobre bloque de goma espuma: hacer como que está dormido o enfermo y que ha de verlo un médico -el adulto-

Coger peluches en brazos (perro y gato), acariciar, hablar, dar de comer, regañar, castigar, pegar.

\subsection{Juegos de precisión}

Introducir pelotas lanzadas dentro de una caja: encestar.

Forcejeos y persecuciones para recuperar y/o quitar objetos que posee el otro.

Realizar carreras con obstáculos colocados en medio de la sala.

Perseguir, pillar, forcejear y escaparse. Lucha de fuerzas.

Lanzamientos de pelota hacia una dirección determinada. Inicio de turnos.

Dar contenido lúdico a propuestas que se le hacen en la sala: tapar el túnel con tela. Quitar la tela, esconderse y asustar.

Meterse dentro del tonel y pasear por diferentes lugares de la sala. Tapar con telas y jugar a abrir y cerrar la puerta. 


\section{Juegos de representación de José}

En el proceso normal de una sesión de psicomotricidad los juegos manipulativos y perceptivo-cognitivos, que constituyen básicamente las actividades de representación, se realizan en la última parte de la sesión. Una vez que el niño ha tenido la oportunidad de experimentar con su cuerpo y sus emociones se proponen actividades encaminadas a tomar distancia de las vivencias sensoriomotoras, de contenido simbólico y afectivo. Serán juegos específicos que, de acuerdo con Arnaiz y Lozano $(1996,36)$, «...son el resultado de toda la movilización anterior por la vía del cuerpo y de las emociones...» y que favorecen que esas experiencias anteriores se transformen en conceptos y en representaciones mentales y que por su estructura didáctica (requieren más calma, más atención, mayor nivel de concentración) y sus contenidos más instrumentales (garabateos, reconocimiento de formas, construcciones con y sin modelo...) sirven de puente en el acceso a contextos escolares.

Estos juegos cumplen, pues, tres funciones principales:

1) Permitir al niño distanciarse de los juegos realizados en otros espacios (juegos de contenido sensoriomotor y simbólico fundamentalmente)

2) Facilitar la descentración afectiva

3) Favorecer el acceso al pensamiento operatorio a partir de procesos de manipulación. experimentación y conceptualización.

\subsection{Juegos manipulativos}

Con la caja de tacos de colores: coger de uno en uno, golpear entre sí, golpear sobre la mesa.

Animar a guardar objetos en caja por orden, de uno en uno y también varios a la vez.

Animar a explorar los elementos nuevos que se le van ofreciendo: pelotas de distintos tamaños, botes y botellas con diferentes cometidos y formas (ranuras y agujeros).

Coger botellas y botes, hacer sonar, abrir y cerrar, volcar, explorar e investigar para sacar lo que hay en su interior. Introducir elementos de uno en uno (pinza lateral, pinza fina).

Pasar objetos de un envase a otro. Introducir cambios en los contenidos de los envases (cubos, bolitas, papeles) así como en el tamaño de los mismos.

Meter anillas pequeñas en eje rígido de madera estando colocado en horizontal y en vertical
Construir torres (2, 3 4, 5 y hasta 6 tacos) con bloques de madera. Tirar y volver a construir.

Explorar nuevos materiales: cartones rugosos, globos, papel de seda, papel de celofán.

Combinar todo tipo de pelotas, teniendo en cuenta la dirección y la fuerza que debe llevar.

Combinar otros elementos: globos...

Patear una pelota, globos.

Golpear con picas sobre la pelota, colchonetas, controlando la fuerza del golpe y sin cometer ninguna agresión.

Enhebrar una bobina en un eje rígido, en alambre $y$ en hilo.

Combinar la pelota o los globos con una pica. Tipo HOCKEY.

Golpear con picas globos lanzados al aire

\subsection{Juegos lógicos básicos y perceptivo-cognitivos}

Buscar objetos que desaparecen ante su mirada. Mantener la trayectoria y recuperarla.

Imitar gestos y ruidos que realizo con la boca, manos y otras partes del cuerpo. Con modelo inmediato y en ausencia de éste.

Estrategias sencillas de recuperación de objetos: esconder bajo una tela, destapar, descubrir; tirar de la cuerda para acercarla; sortear obstáculos para recuperarla.

Señalar e identificar las frutas de plástico. Reconocer tras cambiar el orden en el que son presentadas.

Juegos para ampliar los tiempos en que mantiene la atención: lectura de cuentos, etc.

Actividades que incluyen seguimiento de órdenes sencillas.

Identificar, nombrar, señalar sobre láminas y dibujos sencillos.

Reconocimiento y recuerdo de tonadas y gestos que las identifican.

Seriaciones de elementos de madera o cualquier otro objeto.

Lectura de cuentos de imágenes. Seguir una historia con la mirada, señalar, identificar.

Encajes de figuras geométricas básicas. Propuesta de encajes más complejos.

Presentación de puzzles de dos y tres piezas.

Actividades de papel y lápiz: garabatos y trazos horizontales y verticales. Círculos.

Poner voces diferentes según el objeto-animal de que se trate.

Imitar onomatopeyas que propone el adulto. 


\section{Conclusiones}

Ha interesado en este artículo delimitar y fundamentar el trabajo psicomotor de orientación dinámica que se ha llevado a cabo con un sujeto de 20 meses de edad con síndrome de Down. Se ha elaborado una propuesta de trabajo que recogiese las principales líneas de actuación programadas para las sesiones de psicomotricidad ajustadas a este caso y se han analizado con detalle las diferentes situaciones y actividades lúdicas que se han favorecido dentro del marco de intervención de psicomotricidad propuesto; situaciones relacionadas con los juegos sensoriomotores (situaciones de seguridad profunda, de maternaje, presimbólicas o de contrastes, y sensoriomotoras propiamente dichas); juegos simbólicos (de ficción, de roles y de precisión) y actividades de representación (juegos manipulativos y perceptivocognitivos).

Es interesante reseñar, a modo de conclusión, algunas afirmaciones que se han ido revelando a lo largo de estas páginas:

1) Esta etapa educativa se descubre cercana, pero desconocida a la vez. Ello exige por un lado, de una adecuada formación teórica, pero también requiere de ciertos cambios actitudinales en el adulto; así como de una esmerada renovación de propuestas pedagógicas que permita un mayor conocimiento de las necesidades e intereses de este periodo evolutivo.

2) Se ha mostrado una metodología de trabajo que, con el cuerpo y el movimiento como elementos básicos de expresión del ser humano e instrumento de relación, situándose a partir de las competencias que el sujeto posee y ofreciendo un lugar de seguridad, afectividad y placer, ayude al niño a evolucionar, madurar y adquirir las destrezas físicas (fuerza, precisión...) y psíquicas (identidad, conocimiento de sí mismo...) que le posibiliten el máximo desarrollo de sus competencias. Esta metodología se descubre idónea en el favorecimiento y desarrollo de la actividad lúdica en el niño.

3) Aunque resulta muy difícil demostrar la efectividad real de esta técnica (por la edad del sujeto analizado, por la reducida población con que se ha contado y por otros factores idiosincráticos y de estilo educativo), se han cuantificado mejoras y progresos en la evolución del caso narrado.

4) Se puede afirmar que todos los niños juegan y que este juego es el modo que tienen de descubrir, acercarse y adueñarse del mundo. Las actividades sensoriomotoras, las primeras que le hacen ubicarse en el espacio, en un tiempo y conocerse y probarse a sí mismo, requieren, para un buen desarrollo de las mismas, de una interacción afectiva y motriz que sólo es posible a partir de las situaciones de reaseguración profunda y de maternaje; estrategias lúdicas y de relación indispensables en la conquista del movimiento autónomo y de la acción.

5) La mayor parte del tiempo que han durado las sesiones, la actividad se ha desarrollado en el espacio «sensoriomotor», juegos que comienzan a estar presentes en el momento en que el niño empieza a vivir su cuerpo (3-4 meses). Los juegos simbólicos y de representación han surgido en menor medida y la mayoría de las veces a propuesta del adulto (es importante a este respecto tener presente la temprana edad del sujeto lo que permite ver la imposibilidad evolutiva en el acceso a dichas situaciones).

6) Los objetos que se ponen a disposición del niño en la sala de psicomotricidad facilitan su desarrollo. Es importante que se adecuen a sus necesidades y que no se limiten las posibilidades que el niño les otorga ni los usos que pueda ejercer sobre ellos.

7) Una etapa sensoriomotriz vivida con placer conduce al niño hacia el mundo de la simbolización y más tarde a la representación, al juego social y de reglas de un modo autónomo y seguro. El juego de ficción, al que accede tras el descubrimiento y conformación de sus posibilidades físicas sobre el espacio y los objetos, le permite aventurase en lo prohibido y familiarizarse con los roles sociales de los adultos. Con los juegos de representación categoriza la realidad, pone en orden cuanto le rodea y accede a un conocimiento real acerca del mundo al que pertenece.

\section{Bibliografía}

Arnaiz, P. (2000). La práctica psicomotriz: una estrategia para aprender y comunicar. Revista Iberoamericana de Psicomotricidad y Técnicas Corporales, [en línea], 0, 5-14. Disponible en: http: //www.iberopsicomot.net/num0/0artículo1.pdf [diciembre de 2000]

Arnaiz, P. y Lozano, J. (1996). Proyecto curricular para la diversidad. Psicomotricidad y lectoescritura. Madrid: CCS.

Aucouturier, B., Darrault, I. y Empinet, J. L. (1985). La práctica psicomotriz. Reeducación y terapia. Barcelona: Científico-Médica.

Brunet, O. y Lezine, I. (1971). Le Developpement Psychologique de la première enfance. Paris: PUF (trad. española: Batería de tests para medir el desarrollo psicomotor y la escala de visión de la primera infancia. Madrid: MEPSA).

Llorca, M. y otros (2002). La práctica psicomotriz: una propuesta educativa mediante el cuerpo y el movimiento. Archidona: Aljibe. 